Original Research Paper

\title{
The Effects of Oil Film Shape on Piston Ring and Liner Tribology Under Mixed Lubrication
}

\author{
${ }^{1}$ Abbas Razavykia, ${ }^{2}$ Cristiana Delprete, ${ }^{3}$ Eugenio Brusa and ${ }^{4}$ Yaser Hosseini \\ 1,2,3 Department of Mechanical and Aerospace Engineering, Politecnico di Torino, 10129 Torino, Italy \\ ${ }^{4}$ Department of Engineering, Bahman Diesel Company, Iran
}

\author{
Article history \\ Received: 23-09-2021 \\ Revised: $10-10-2021$ \\ Accepted: 15-10-2021 \\ Corresponding Author: \\ Abbas Razavykia \\ Department of Mechanical and \\ Aerospace Engineering, \\ Politecnico di Torino, 10129 \\ Torino, Italy \\ Email: abbas.razavykia@polito.it
}

\begin{abstract}
Mechanical power loss reduction at lubricated reciprocating and rotating components is recognized as an approach to improve the efficiency and to reduce the emissions of Internal Combustion Engines (ICEs). To achieve these goals, the instantaneous investigation of lubrication characteristics is required. Piston ring pack is of paramount importance as it is known as major contributor to frictional losses and energy dissipation. Applying Reynolds equation and lubrication theory to study piston ring tribology, requires specifying of boundary conditions. Oil film characteristics (shape and thickness) and generated hydrodynamic pressure are under influence of considered boundary conditions. Besides, the type of selected boundary conditions affects analysis robustness and sensitivity. During engine strokes, piston ring enjoys hydrodynamic and mixed lubrication regimes. The principle aim of the current study is to examine the effects of alternative boundary conditions: Half Sommerfeld, oil separation and Reynolds cavitation and reformation conditions on piston ring tribology under isothermal mixed and hydrodynamic lubrication regimes. This article demonstrates that different boundary conditions are suited to different operating conditions with respect to load, speed and temperature as well as crank angle, i.e., relative position of ring with respect to the liner. Thicker oil film thickness has been calculated applying half Sommerfeld boundary conditions under either hydrodynamic or mixed lubrication regimes followed by oil separation due to larger effective of the ring width. It was observed that considering oil separation boundary conditions results in lower deviation from experimental data, followed by Sommerfeld boundary conditions under mixed lubrication.
\end{abstract}

Keywords: Internal Combustion Engine, Reynolds Equation, Hydrodynamic, Mixed Lubrication, Sommerfeld, Cavitation, Piston Ring

\section{Introduction}

In contrary to experimental investigation of piston ring/liner interface lubrication, which is time consuming, costly and varies from case to case, analytical formulation and numerical simulation might be a reliable alternative. Development of robust lubrication models would direct designers and engineers to realize, where the modification can be done to Improve Internal Combustion Engines (ICEs) efficiency in terms of oil and fuel consumption as well as emissions (Delprete and Razavykia, 2018). Applying lubrication theory and considering Reynolds equation to analyze piston ring lubrication with parabolic axial ring profile, specifying boundary conditions is essential. The nature of generated hydrodynamic pressure at interfaces, Load Carrying Capacity (LCC) and oil film thickness are under influence of employed boundary conditions (Priest et al., 1996).

A vast number of researchers consider different boundary conditions to solve Reynolds equation and analyse piston ring tribology. It is well known that the dominant lubrication regime during engine strokes is the mixed one, in particular at critical regions, top and Bottom Dead Centers (TDC and BDC), followed by hydrodynamic lubrication with respect to operating conditions, loads and temperature. Obtaining deep insight into transient nature of ring/piston contact and lubrication serves as a prelude to understand contact kinematics and bearing couple conformance. Dowson et al. (1983) examined piston ring lubrication subjected to elasto-hydrodynamic lubrication, in 
which film rupture takes place when hydrodynamic pressure descends below the atmospheric pressure, which can be governed by Reynolds boundary conditions. Fully flooded condition was compared against starved lubrication in terms of obtained minimum oil film thickness. Thicker oil film was calculated under fully flooded due to wedge action at midphase of engine strokes (Jeng, 1992). Reynolds boundary conditions were found to be fairly acceptable and in good agreement with experimental data especially in critical regions where the squeeze film effect dominates and there would be no oil rupture and cavitation (Chu-Jung and Cheng, 1991; Richardson and Borman, 1992; Cho et al., 2000; Akalin and Newaz, 2001).

Cavitation boundary and contact exit boundary conditions have been applied to examine piston ring lubrication mechanisms. It was highlighted that the hydrodynamic pressure profiles, lubricant film boundaries and thickness, oil flow and frictional losses are under significant influence of considered boundary conditions (Priest et al., 2000).

Han and Lee (1998) applied oil starvation and the open-end boundary conditions; it was observed that effective ring width reduced by 20 to 30 percent, but entire ring width comes into the contact under mixed lubrication due to the dominant squeeze effect. The effects of fully flooded and starved boundary conditions on oil film thickness and frictional loss have been studied. It was observed that at TDC and BDC, cavitation is limited by squeeze action (Livanos and Kyrtatos, 2006). Morris et al. (2013b) applied oil separation boundary conditions to study piston ring geometry and its face topography under mixed lubrication and later ring/liner contact behavior using a new analytical thermal model (Morris et al., 2013a). A mixed lubrication considering oil supply quantity has been developed. It was concluded that under fully flooded condition, the obtained results, (Greenwood and Tripp, 1970) and experimental data are in accordance with each other except in TDC and BDC. In contrast, under starved condition, model is closer to experimental data rather than Greenwood-Tripp model in the entire stroke (Guo et al., 2015). The effect of cylinder liner temperature on the lubrication and friction loss at the ring and liner conjunction was investigated. The Swift-Stieber exit boundary conditions have been used to determine the lubricant film rupture and the cavitation region. It was assumed that the pressure throughout the cavitation region remains constant and is equal to the ambient pressure (Rahmani et al., 2017).

The principle aims of the current study are to examine alternative boundary conditions that can be applied during numerical simulation of the piston ring/liner tribological performance and to evaluate the solution sensitivity to these boundary conditions. Comprehensive formulation has been introduced to compare the impact of half Sommerfeld, oil separation and Reynolds cavitation and reformation boundary conditions on analysis sensitivity under mixed and hydrodynamic lubrication regimes of ring and cylinder wall junction. The scheme presented in this investigation enables the depiction of the transition between various level of ring face engulfment, fully, partially flooded and cavitated. The results have been compared against experimental data to encourage accurate selection of boundary conditions.

\section{Model and Procedure}

Considering circumferential symmetry of oil distribution at ring and liner interface, for the ring those length exceeding their axial face-width by a factor of 30, onedimensional Reynolds equation can be applied to evaluate the ring and liner tribological performance (Morris et al., $2013 b$ ). For an isothermal and incompressible lubricant, 1D-Reynolds equation can be written as:

$\frac{\partial}{\partial x}\left(\frac{h_{T}^{3}}{12 \eta} \frac{\partial p}{\partial x}\right)=\frac{U_{1}+U_{2}}{2} \frac{\partial h_{T}}{\partial x}+\frac{\partial h_{T}}{\partial t}$

where, $x$ is the coordinate parallel to bore axis, $h_{T}$ the average gap or rough surface film thickness, $\eta$ the lubricant dynamic viscosity $(\mathrm{Pa} \cdot \mathrm{s}), U_{1}$ and $U_{2}$ the ring and liner sliding speeds $(\mathrm{m} / \mathrm{s})$ respectively and $t$ the time (s).

The local film thickness, $h_{T}$, at ring and liner junction considering surfaces roughness profiles, $\delta_{1}$ and $\delta_{2}$ respectively as shown by Fig. 1 (a), is expressed as:

$h_{T}(x, t)=h(x, t)+\partial_{1}+\partial_{2}$

where, $h(x, t)$ is the nominal film thickness as a function of time (or crank angle).

Regarding to Fig. 1 (b), nominal oil film thickness can be defined using:

$$
\begin{aligned}
& h(x, t)=h_{\text {min }}(t)+\frac{c}{\left(\frac{b}{2}+o\right)^{2}}(x-o)^{2}= \\
& =h_{\text {min }}(t)+B(x-o)^{2}
\end{aligned}
$$

where, $h_{\min }$ is the minimum oil film thickness, $c$ the ring crown height, $b$ the ring axial width, o the ring offset and $B=c /(b / 2+o)^{2}$ the ring profile curvature.

It is assumed that asperities follow Gaussian distribution with mean of zero and standard deviations $\sigma_{1}$ and $\sigma_{2}$. Equivalent value of roughness amplitudes can be represented by the RMS of combined surface roughness $\sigma=\sqrt{\sigma_{1}^{2}+\sigma_{2}^{2}}$. 
(a)

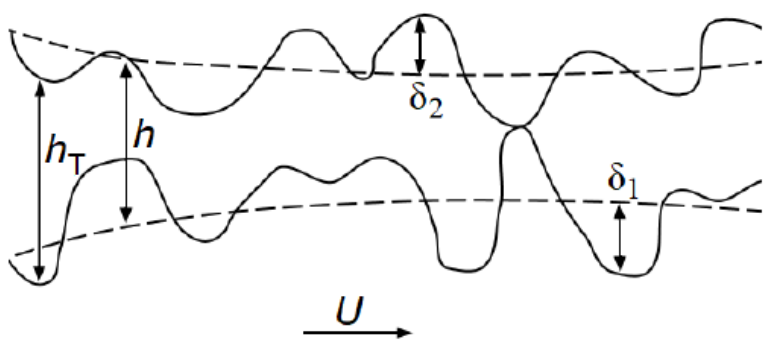

(b)

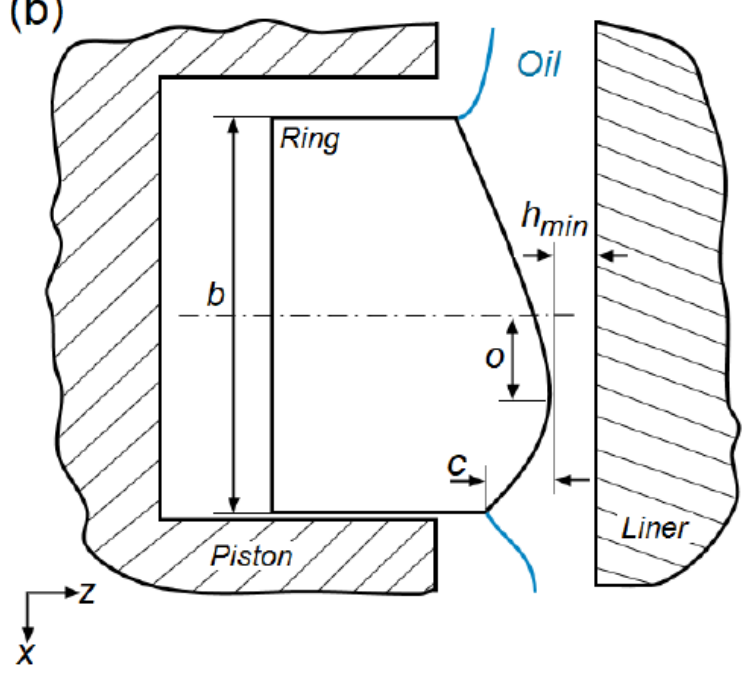

Fig. 1: (a) Oil film thickness function and (b) Nominal clearance at ring and liner junction

Substituting Eq. (2) in (1) and considering the contact factor $\varphi_{c}=d h_{T} / d h$, the average Reynolds equation modified by Patir and Cheng (1979) in terms of pressure and shear flow factors, which are functions of surface roughness, can be written as:

$$
\begin{aligned}
& \frac{\partial}{\partial x}\left(\phi_{x} \frac{h^{3}}{12 \eta} \frac{\partial \bar{p}}{\partial x}\right)=\frac{U_{1}+U_{2}}{2} \phi_{c} \frac{\partial h}{\partial x}+ \\
& +\frac{\Delta U}{2} \sigma \frac{\partial \phi_{s}}{\partial x}+\phi_{c} \frac{\partial h}{\partial t}
\end{aligned}
$$

where, $\Delta U=U_{1}-U_{2}, \varphi_{x}$ is the pressure flow factor and $\varphi_{s}$ the shear flow factor along the cylinder axis.

Ignoring fluttering and tilting motions of the ring in the groove, due to unbalanced forces, sliding speed as same as piston reciprocating speed can be considered for the ring and is calculated as:

$$
U=\omega r\left[\sin \theta-\frac{\cos \theta(\Lambda \sin \theta-\delta)}{\sqrt{1-(\Lambda \sin \theta-\delta)^{2}}}\right]
$$

where, $\omega$ is the engine spin speed $(\mathrm{rad} / \mathrm{s}), \theta$ the crank angle, $r$ the crank radius $(\mathrm{m}), \Lambda=r / l$ the elongation ratio and $\delta$ the nondimensional offset $\delta=z_{o} / 1$, with $z_{o}$ the overall offset (crankshaft offset $z_{o c}+$ wrist pin offset $z_{o w p}$ and 1 the connecting rod lenght).

In order to determine the coordinate system on the ring axial face, ring is assumed to be stationary $\left(U_{1}=0\right)$ and the liner is sliding against the ring with same speed of piston but in opposite direction, $U_{2}=-U$. Therefore, Eq. (4) can be written as:

$\frac{\partial}{\partial x}\left(\phi_{x} \frac{h^{3}}{12 \eta} \frac{\partial \bar{p}}{\partial x}\right)=-\frac{U}{2} \phi_{c} \frac{\partial h}{\partial x}+\frac{U}{2} \sigma \frac{\partial \phi_{s}}{\partial x}+\phi_{c} \frac{\partial h}{\partial t}$

Considering an iso-viscous incompressible lubricant and based on Patir and Cheng average flow model (Patir and Cheng, 1979), pressure flow factor $\varphi_{x}$ and shear flow factor $\varphi_{s}$ are functions of local film thickness parameter to composite surface roughness ratio $(H=h / \sigma)$ and surface roughness orientation factor $\gamma$.

Pressure flow factor, $\varphi_{x}$, compares the average pressure flow under rough surface to that of a smooth surface, which is determined as:

$\phi_{x}= \begin{cases}1-C e^{-k H} & \gamma \leq 1 \\ 1+C e^{-k} & \gamma>1\end{cases}$

Assuming $\mathrm{H}>0.5$ and an isotropic roughness $(\gamma=1)$, then $\mathrm{C}=0.9, \mathrm{k}=0.56$ and $\varphi_{x}=1-0.9 \mathrm{e}^{-0.56 \mathrm{H}}$.

Assuming $\varphi_{\mathrm{s}}$ as representation of an additional flow due to rough bearing surface sliding, it is calculated as:

$\phi_{x}= \begin{cases}A_{1} H^{\alpha_{1}}\left(V_{1}-V_{2}\right) e^{\left(-\alpha_{2} H+\alpha_{3} H^{2}\right)} H \leq 5 \\ A_{2}\left(V_{1}-V_{2}\right) e^{-0.25 H} & H>5\end{cases}$

where, $\mathrm{V}_{1}$ and $\mathrm{V}_{2}$ are the variance ratios $V_{i}=\frac{\sigma_{i}^{2}}{\sigma^{2}}, i \in 1,2$ (Patir and Cheng, 1979; Mishra et al., 2009).

Equations (4) and (6) alter to Reynolds equation for smooth surface if $\varphi_{x}, \varphi_{s}$ and $H$ satisfy:

$\phi_{x} \rightarrow 1$ and $\phi_{s} \rightarrow 0$ as $H \rightarrow \infty$

For an isotropic roughness $(\mathrm{H}>0.5)$, coefficients for shear flow factor are tabulated by Table 1 (Patir and Cheng, 1979).

Table 1: Coefficients for shear flow factor

\begin{tabular}{llllll}
\hline$\gamma$ & $\mathrm{A}_{1}$ & $\alpha 1$ & $\alpha 2$ & $\alpha 3$ & $\mathrm{~A}_{2}$ \\
\hline 1 & 1.899 & 0.98 & 0.92 & 0.05 & 1.126 \\
\hline
\end{tabular}


Assuming the Gaussian distribution with unit variance and zero mean for asperities heights, the standard probability density function is:

$\varphi(s)=\frac{1}{\sqrt{2 \pi}} e^{-\frac{s^{2}}{2}},-\infty<s<\infty$

and contact factor for mixed lubrication of ring and liner junction (Wu and Zheng, 1989) is:

$$
\phi_{c}=\int_{H}^{\infty} \varphi(s) d s=\frac{1}{2}[1+\operatorname{erf}(H)]
$$

Two times integration of Eq. (6) with respect to $x$, gives the average pressure contributed by generated hydrodynamic and asperities contact pressures at ring and liner junction:

$$
\begin{aligned}
& \bar{p}=-\frac{6 \eta U}{\phi_{x}} \underbrace{\int_{x}^{\frac{h \phi_{c}-\sigma \phi_{s}}{h_{3}} d x}}_{I_{0}(x)}+ \\
& +\frac{12 \eta \phi_{c} \omega}{\phi_{x}} \frac{\partial h}{\partial \theta} \underbrace{\frac{x}{h^{3}} d x}_{I_{1}(x)}+\frac{C_{1}}{\phi_{x}} \underbrace{\int_{x}^{\frac{1}{h^{3}} d x+C_{2}}}_{I_{2}(x)}
\end{aligned}
$$

where, $C_{1}$ and $C_{2}$ are the integration constants and

$$
\begin{aligned}
& \left.I_{0}(x)=\phi_{c}\left(\frac{\tan ^{-1}\left[\sqrt{\frac{B}{h_{\min }}(x-o)}\right]}{2 h_{\min } \sqrt{B h_{\min }}}+\frac{(x-o)}{2 h_{\min }\left[h_{\min }+B(x-o)^{2}\right]}\right)\right) \\
& +-\sigma \phi_{s}\left(\frac{3 \tan ^{-1}\left[\sqrt{\frac{B}{h_{\min }}}(x-o)\right]}{8 h_{\min }^{2} \sqrt{B h_{\min }}}++\frac{(x-o)\left[3 B(x-o)^{2}+5 h_{\min }\right]}{8 h_{\min }^{2}\left[h_{\min }+B(x-o)^{2}\right]^{2}}\right) \\
& I_{1}(x)=\frac{3 o \tan ^{-1}\left[\sqrt{\frac{B}{h_{\min }}(x-o)}\right]}{8 h_{\min }^{2} \sqrt{B h_{\min }}}+\frac{3 o(x-o)}{8 h_{\min }^{2}\left[h_{\min }+B(x-o)^{2}\right]}+ \\
& +\frac{o(x-o)}{4 h_{\min }\left[h_{\min }+B(x-o)^{2}\right]^{2}}+-\frac{1}{4 B\left[h_{\min }+B(x-o)^{2}\right]^{2}} \\
& I_{2}(x)=\frac{3 \tan ^{-1}\left[\sqrt{\frac{B}{h_{\min }}(x-o)}\right]}{8 h_{\min }^{2} \sqrt{B h_{\min }}}+ \\
& +\frac{(x-o)\left[3 B(x-o)^{2}+5 h_{\min }\right]}{8 h_{\min }^{2}\left[h_{\min }+B(x-o)^{2}\right]^{2}}
\end{aligned}
$$

Average force acting on ring face contributed by the generated hydrodynamic pressure and asperities contact under mixed lubrication is obtained via integration of Eq. (12) with respect to $x$ :

$$
\begin{aligned}
& F_{h, c}(x)=-\frac{6 \eta U}{\phi_{x}} J_{0}(x)+\frac{12 \eta \phi_{c} \omega}{\phi_{x}} \frac{d h}{d \theta} J_{1}(x)+ \\
& +\frac{C_{1}}{\phi_{x}} J_{2}(x)+C_{2} x
\end{aligned}
$$

where:

$$
\begin{aligned}
& J_{0}(x)=\phi_{c}\left(\frac{(x-o) \tan ^{-1}\left[\sqrt{\frac{B}{h_{\text {min }}}(x-o)}\right]}{2 h_{\min } \sqrt{B h_{\min }}}\right)+ \\
& -\sigma \phi_{s}\left(\frac{3(x-o) \tan ^{-1}\left[\sqrt{\frac{B}{h_{\min }}(x-o)}\right]}{8 h_{\min }^{2} \sqrt{B h_{\min }}}+-\frac{1}{8 B h_{\min }\left[h_{\min }+B(x-o)^{2}\right]}\right) \\
& J_{1}(x)=\frac{\left(3 B o(x-o)-h_{\min }\right) \tan ^{-1}\left[\sqrt{\frac{B}{h_{\text {min }}}(x-o)}\right]}{8 B h_{\min }^{2} \sqrt{B h_{\min }}}+ \\
& -\frac{x}{8 B h_{\min }\left[h_{\min }+B(x-o)^{2}\right]} \\
& J_{2}(x)=\frac{3(x-o) \tan ^{-1}\left[\sqrt{\frac{B}{h_{\min }}(x-o)}\right]}{8 B h_{\min }^{2} \sqrt{B h_{\min }}}+ \\
& -\frac{x}{8 B h_{\min }\left[h_{\min }+B(x-o)^{2}\right]}
\end{aligned}
$$

Sommerfeld, oil separation and Reynolds cavitation and reformation boundary conditions, have been applied to calculate the integration constants $C_{1}$ and $C_{2}$ and to examine their effects.

Full Sommerfeld boundary condition is the simplest solution of Reynolds equation considering no oil film rupture. Unrealistically, applying full Sommerfeld solution imposes the fluid to continuously carry significant negative pressures and obtain underestimation of the lubricant LCC. These errors were solved by Gumbel, by" proposing that generated negative pressure easily must be ignored and assumed to be equal to boundary pressure acting on the trailing edge of the ring, which is known as half Sommerfeld condition (Priest et al., 1996). Therefore, considering half Sommerfeld boundary conditions, as shown by Fig. 2(a), gas pressures acting on upper and lower edges of the ring, $p_{u p}$ and $p_{\text {down }}$ respectively, are the only boundary conditions those are defined in downward stroke as: 


$$
\begin{array}{cc}
p=p_{\text {down }}, & x=x_{\text {in }} \\
p=p_{\text {up }}, & x=x_{e x}
\end{array}
$$

where, $x_{i n}$ is the point that lubricant touches ring leading edge and $x_{e x}$ the point that lubricant leaves ring trailing edge.

Applying force balance acting on the ring, the load contributed by ring elastic and gas pressures, $F_{\text {ring,gas }}$ is in equilibrium with the average force acting on the ring face, $F_{h, c}$, as the resultant force due to hydrodynamic and asperities average contact pressures, $p_{h}$ and $p_{a}$, respectively. The problem can be solved as quasi-steadystate problem in which:

$$
F_{h, c}(\theta)-F_{\text {ring, gas }}(\theta)=0
$$

with:

$$
F_{\text {ring,gas }}(\theta)=b\left(p_{\text {ring }}+p_{\text {gas }}\right)=b\left(\frac{2 T}{b D}+p_{\text {gas }}\right)
$$

where, $T$ is the ring tension force, $D$ the cylinder bore diameter and $p_{\text {gas }}$ gas pressure over the ring back side, which is the maximum of boundary pressures acting on the ring upper and lower edges. Therefore, force balance equation applying half Sommerfeld boundary conditions, in downward stroke can be expressed as:

$$
\begin{aligned}
& \int_{o}^{x_{i n}} p(x) d x+\left(a-x_{\text {in }}\right) p_{\text {down }}+(a+o) p_{\text {up }}+ \\
& +\int_{-\frac{B}{2}}^{\frac{B}{2}} p_{a}(x) d x=b\left(p_{\text {back }}+\frac{2 T}{b D}\right)
\end{aligned}
$$

where a is half of the ring axial width.

Average contact pressure at ring and liner interface due to opposing asperities contacts can be defined as:

$$
p_{a}(x)=\frac{8 \sqrt{2}}{15} \pi(\xi K \sigma)^{2} \sqrt{\frac{\sigma}{K}} E^{\prime} A F_{2.5}(H)
$$

with:

$$
F_{2.5}(H)=\frac{1}{2 \pi} \int_{H}^{\infty}(s-H)^{2.5} e^{\frac{s^{2}}{2}} d s
$$

where, $\zeta$ is the asperity density per unit contact area, $K$ the average asperity tip radius, ${ }^{E 0}$ the reduced (effective) elastic modulus of the contacting pair, The apparent contact area and $\mathrm{s}$ the profile of ring face-width.

The more convenient form of Eq. (25), can be expressed as:

$$
F_{2.5}(H) \begin{cases}A(\Omega-H)^{z} & H \leq \Omega \\ 0 & H>\Omega\end{cases}
$$

where, $\Omega=4.0, \mathrm{~A}=4.4068 \mathrm{e}-05, \mathrm{z}=6.804$ and $\mathrm{K}=$ 1.198e-04 (Akalin and Newaz, 2001). Regarding to upward stroke, boundary conditions and force equilibrium can be defined in the same manner.

Considering laminar flow of the lubricant, oil layers are swept and accumulated in front of ring leading edge, as consequence of ring sliding motion. Therefore, the leading edge is completely engulfed and the trailing edge is partially covered by the oil as illustrated in Fig. 2(b). As result of converging-diverging ring profile as well as the sudden reduction and alteration of hydrodynamic pressure from positive to negative, oil film ruptures and consequently, some area of the trailing edge exposes to boundary gas, which is called separation condition (Delprete et al., 2020). Under this circumstance, the oil film rupture is treated as flow separation rather than cavitation (Richardson and Borman, 1992).

Regarding to Fig. 2 (b), separation boundary conditions can be written in downward stroke as:

$\begin{array}{cc}p=p_{\text {down }}, & x=a \\ p=p_{\text {up }}, \frac{d p}{d x}=0 & x=x_{\text {ex }}\end{array}$

There are three unknowns, $h_{\min }, x_{e}$ and $d h / d \theta$ while applying separation boundary conditions. Therefore, load and pressure equilibrium in downward stroke are expressed as:

$p\left(x_{e}\right)=p_{u p}$

$\int_{x_{e}}^{a} p(x) d x+p_{u p}\left(a-x_{e}\right)+$

$+\int_{-\frac{B}{2}}^{\frac{B}{2}} p a(x) d x=b\left(\frac{2 T}{b D}+\right.$ pgas $)$

same approach can be adopted in case of upward stroke.

Reynolds cavitation and reformation boundary conditions are shown in Fig. 2(c). Under this condition, gas bubbles are entrapping within oil film. The pressure in the gas cavities is treated to be equal to saturation pressure (atmospheric). Oil flow continuity across the cavity, convinced the determination of pressure radiant at reformation point. Reynolds cavitation and reformation boundary conditions. 

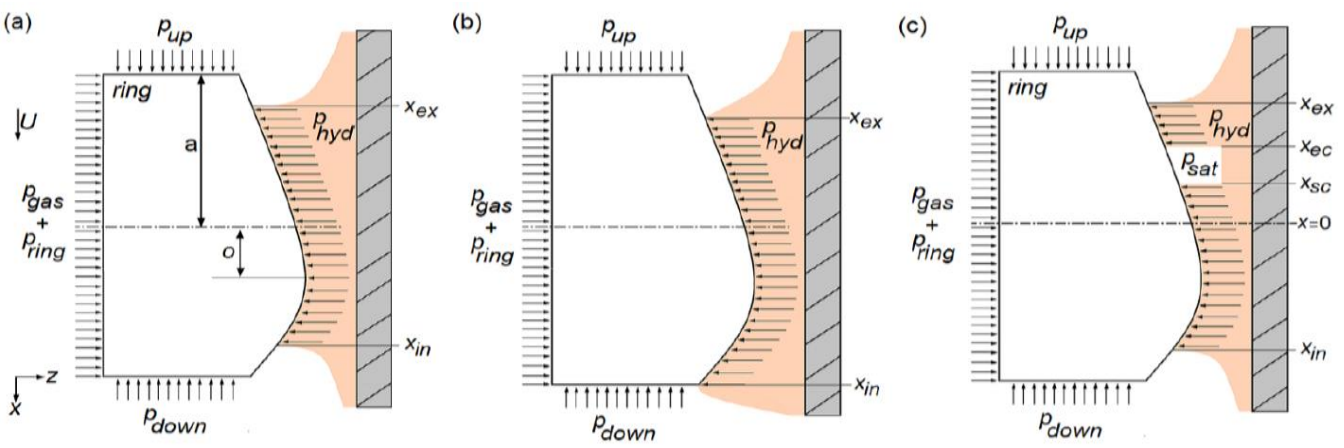

Fig. 2: Applied boundary conditions: (a) Half Sommerfeld, (b) oil separation and (c) Reynolds cavitation and reformation boundary conditions

boundary conditions in downward stroke are expressed as:

$$
\begin{array}{ll}
p=p_{\text {down }} & x=x_{\text {in }} \\
p=0 \frac{d p}{d x}=0 & x=x_{s c} \\
p=0, \frac{d p}{d x}=6 \eta U \frac{h(x e c, \theta)-h(x s c, \theta)}{h^{3}(x e c, \theta)} & x=x e c \\
p=p_{u p} & x=x_{e x}
\end{array}
$$

where, $x_{s c}$ is the cavity start point and $x_{e c}$ the cavity end point.

To calculate the instantaneous minimum oil film thickness $h_{\min }$ and the oil boundary points $x_{i n}, x_{s c}, x_{e c}$ and $x_{e x}$ in downward stroke, a system of five equations must be solved:

$$
\begin{aligned}
& \int_{x_{i n}}^{x_{s c}} p(x) d x+\int_{x_{e c}}^{x_{e x}} p(x) d x+\text { pdown }(b-x i n) \\
& +p_{u p}\left(b-x_{e x}\right)+\int_{-\frac{B}{2}}^{F B} p_{a}(x) d x=b\left(\frac{2 T}{b D}+p_{\text {gas }}\right) \\
& p\left(x_{\text {in }}=p_{\text {down }}\right) \\
& p\left(x_{s c}\right)=\frac{d p}{d x}\left(x_{s c}\right)=0 \\
& p\left(x_{e c}\right)=0, \frac{d p}{d x}\left(x_{e c}\right)=6 \eta U\left(\frac{h\left(x_{e c}, \theta\right)-h\left(x_{s c}, \theta\right)}{h^{3}\left(x_{e c}, \theta\right)}\right) \\
& p\left(x_{e x}\right)=p_{u p}
\end{aligned}
$$

and corresponding governing equation in upward stroke can be written in the same manner.

\section{Numerical Solution for Ring Pack Lubrication Analysis}

Regarding to aforementioned boundary conditions and shortage of one equation with respect to the number of unknowns, applying numerical scheme is required to solve the problem. Considering half Sommerfeld boundary conditions which is the most simplest case in comparison to the others, there are two unknowns $h_{\min }$ and $\partial \mathrm{h} / \partial \theta$ and one governing equation (Eq. 23).

Figure 3 shows the algorithm and iterative method to solve the problem under steady state condition. Time step can be defined as crank angle instead of time using $\theta=\omega t$. Starting from mid phase of the engine stroke where hydrodynamic lubrication is dominated and $\partial \mathrm{h} / \partial \theta$ is expected to change slightly, therefore an estimation of $h_{\text {min }}$ can be made. Therefore, the term of $\partial \mathrm{h} / \partial \theta$ can be neglected and Eq. 23 can be solved applying to calculate minimum oil film thickness, $h_{\min }$. At the next angle, all the operating conditions data should be updated and the calculated $h_{\min }$ in previous crank angle is used to have estimation of $\partial \mathrm{h} / \partial \theta$ and determine film thickness. This iterative approach and employing Newton-Raphson method encourages the determination of minimum oil film thickness at ring and liner junction for whole engine cycle.

\section{Friction Losses}

Friction losses under mixed lubrication are contributed by shearing of asperities and viscous shear stress within oil film thickness. Based on Patir and Cheng (1979) average flow model, viscous shear stress is a function of flow factors that they are dependent of oil film parameter, $\mathrm{H}$ (Morris et al., 2013b; Patir and Cheng, 1979). Hence, viscous friction force becomes:

$f_{v}=2 \pi R \int_{x}\left(\phi_{f p} \frac{h}{2} \frac{\partial \bar{p}}{\partial x} \pm\left(\phi_{f} \pm \phi_{f s}\right) \frac{\eta \Delta U}{h}\right) d x$

The shear stress factors $\varphi_{\mathrm{f}}, \varphi_{\mathrm{fs}}$ and $\varphi_{\mathrm{f}}$ are calculated as (Patir and Cheng, 1979):

$\phi_{f p}=1-1.4 e^{-0.66 H}, H>0.75, \gamma=1$ 


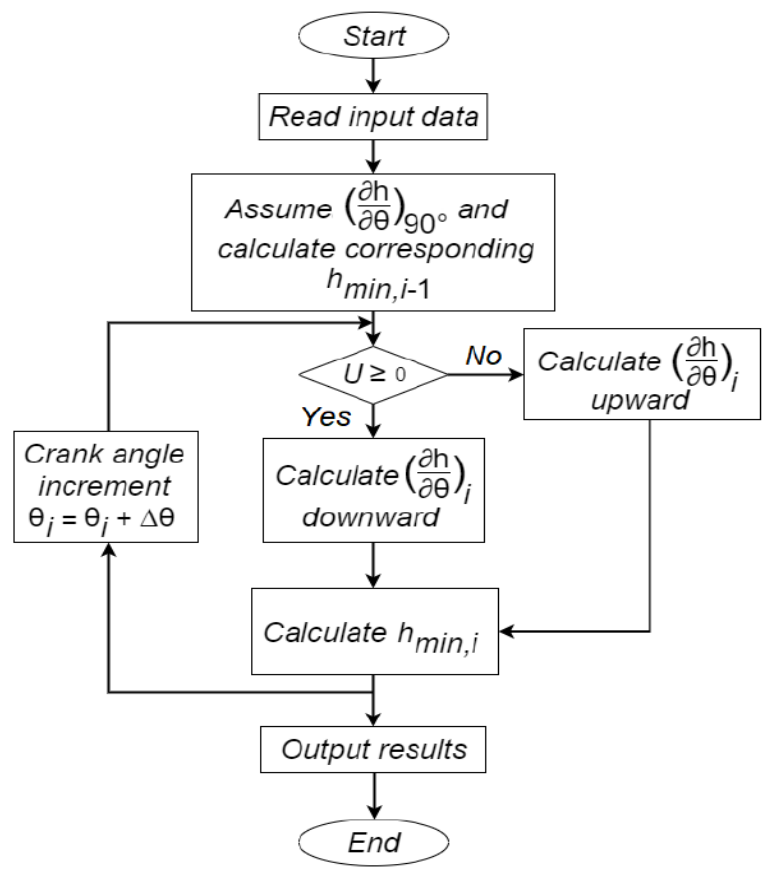

Fig. 3: Solution flow chart

Table 2: Relevant engine parameters

\begin{tabular}{ll}
\hline$b$ & ring width $1.5 \mathrm{~mm}$ \\
$c$ & crown height $12 \mathrm{~mm}$ \\
$D$ & cylinder bore $83.80 \mathrm{~mm}$ \\
$E$ & composite Young's modulus $1.1379 \mathrm{GPa}$ \\
$l$ & connecting rod length $153 \mathrm{~mm}$ \\
$o$ & ring offset $0.0 \mathrm{~mm}$ \\
$r$ & crank radius $49.5 \mathrm{~mm}$ \\
$T$ & ring elastic tension $10 \mathrm{~N}$ \\
$Z_{\text {owp }}$ & wrist pin offset $0.5 \mathrm{~mm}$ \\
$Z_{o c}$ & crankshaft offset $0.0 \mathrm{~mm}$ \\
$h$ & oil dynamic viscosity $11.73 \mathrm{E}-03 \mathrm{~Pa} \_\mathrm{s}$ \\
$s$ & combined surface roughness $0.37 \mathrm{~mm}$ \\
\hline
\end{tabular}

$\phi_{f s}=\left\{\begin{array}{c}11.1 H^{2.31} e\left(-2.38 H+0.11 H^{2}\right) 0.5<H<7, \gamma=1 \\ 0 \quad H>7, \gamma=1\end{array}\right.$

and:

$\phi_{f}\left\{\begin{array}{cc}\frac{35}{32} \xi\left(\left(1-\xi^{2}\right)^{3} \ln [300(1+\xi)]+\frac{1}{60} N\right) & H \leq 3 \\ \frac{35}{32} \xi\left(\left(1-\xi^{2}\right)^{3} \ln \left(\frac{\xi+1}{\xi-1}\right)+\frac{\xi}{15}\left[66+\xi^{2}\left(30 \xi^{2}-80\right)\right]\right) & H>3\end{array}\right.$

where, $\xi=H / 3$,

$N=\xi(\xi[132+\xi(345+M)])-55$

and:
$M=\xi(\xi[\xi(\xi 60+147 \xi)-405]-160)$

The role of mixed lubrication becomes more significant at least in some critical regions such as TDC and BDC, where there is momentary cessation of oil-entraining flow. Thus, friction force due to asperities contact can be calculated as:

$$
f_{a}=\mu_{f} \int_{-\frac{B}{2}}^{\frac{B}{2}} p a(x) d x
$$

where, $\mu_{f}$ is the dynamic asperity friction coefficient. Therefore, total frictional loss at ring and liner interface is obtained:

$$
f_{f}=f_{v}+f_{a}
$$

\section{Experimental Validation}

Strip down approach has been applied on a $2.2 \mathrm{~L}$ cast iron $200 \mathrm{hp}$ engine in which lubricant fluid was supplied to the lubricated parts junction using an external pump. The temperature of lubricant and cooling fluids kept unchanged by means of utilizing separated external circuits. Engine spin speed varied from 1000 to 4000, in order to evaluate different operating conditions.

The Friction Means Effective Pressure (FMEP) measurement of the Piston Ring Assembly (PRA) was carried out at four steps. First, the FMEP was calculated for the cranktrain including crankshaft and piston ring assembly. At the second step, measuring FMEP for crankshaft and then for third part, piston group FMEP were recorded. Finally, FMEP for piston ring were defined and converted to mean power loss (W) to be compared with the obtained data from the model.

Relevant data for engine and compression ring assembly are tabulated by Table 2 .

\section{Results and Discussion}

Inter ring gas pressure (blow-by) for a ring pack including two compression rings and one oil control ring with two rails, at engine speed of $2000 \mathrm{rpm}$ is shown in Fig. 4. Figure 5 compares the minimum oil film thickness under hydrodynamic and mixed lubrication at spin speed of $2000 \mathrm{rpm}$, applying half Sommerfeld boundary conditions in which ring face is engulfed by lubricant and there is no oil film rupture. Detailed analysis of piston ring tribology under hydrodynamic lubrication using half Sommerfeld, oil separation and Reynolds cavitation and reformation boundary conditions, has been reported in (Delprete et al., 2020). Mostly, higher minimum oil film thickness has been calculated under hydrodynamic regime in comparison to mixed lubrication, as the imposed normal load contributed by ring elastic tension and the gas pressure acting on the ring back side, should be sustained only by the generated 
hydrodynamic pressure at ring and liner interface (?). Therefore, the thicker oil film thickness under hydrodynamic regime is achieved to guarantee the creation of adequate hydrodynamic pressure to lift the ring and to obtain smoother reciprocating motion of the ring against liner. The generated hydrodynamic lubrication at ring and liner junction is due to the shear stress within the film is proportional to the film thickness. Considering laminar flow of the lubricant layers, the higher numbers of them is demanded to stand against the imposed normal load until certain magnitude (fluid LCC) at the given sliding speed, so thicker minimum film thickness under hydrodynamic lubrication is expected. Higher friction force has been calculated considering mixed lubrication at ring and liner junction in comparison to hydrodynamic regime. In critical regions, TDC and BDC, due to lower piston sliding velocity and in particular high temperature in TDC, asperities contact and surface flow factors play important roles and frictional losses mostly is contributed by contact pressure Eq. (24). Lubricant LCC is very sensitive to temperature, as oil viscosity drops with temperature increment; in this circumstance, the load is sustained mostly by contact asperities. The deviation of frictional loss under mixed and lubrication regime at $2000 \mathrm{rpm}$ is shown in Fig. 6 considering half Sommerfeld boundary conditions. Figure 7 demonstrates the engine speed impact on frictional losses at ring/liner interface under mixed lubrication regime applying half Sommerfeld boundary conditions; the higher the engine speed, the more the shear stress and in consequence larger friction force.

Considering oil separation boundary conditions under hydrodynamic, thicker $h_{m}$ has been calculated in comparison to mixed regime, as the load should be sustained just by hydrodynamic pressure, as shown by Fig. 8. Even, under same lubrication regime, either hydrodynamic or mixed, applying oil separation conditions results in thicker calculated $h_{m}$ as effective width of ring is shorter than fully flooded condition, in which whole ring width comes in contact by lubricant (Taylor, 2015). Applying oil separation conditions, larger frictional losses in most time instance has been calculated under mixed lubrication, as Fig. 9 compares both lubrication regimes at $2000 \mathrm{rpm}$ of engine spin speed. Transition of hydrodynamic to mixed lubrication and vice versa during the engine strokes, is strongly sensitive to the load (gas pressure), engine spin speed and temperature. The lower the spin speed, the larger the contact area and in turn, the higher the contact resistance and power loss (Hu and Zhu, 2000). At higher temperature, due to lubricant LCC reduction, the hydrodynamic effect vanishes. Therefore, regarding to regular engine operating conditions, mixed lubrication is dominated in most time instance during the strokes, in particular compression and expansion. Figure 10 illustrates the engine spin speed on frictional loss under mixed lubrication applying separation boundary conditions. Comparing Fig. 7 and 10, lower friction force due the viscous shear stress has been obtained considering oil separation conditions in most crank angles as the thinner film thickness has been calculated in comparison to half Sommerfeld conditions. In contrast, in TDC and $\mathrm{BDC}$, higher frictional loss has been calculated compared to applying Sommerfeld boundary conditions.

Figure 11 shows ring axial profiles for different crown height, under mixed lubrication regime. Thicker minimum oil film thickness was calculated for the crown height of 9 mm applying separation boundary condition at $2000 \mathrm{rpm}$, as shown by Fig. 12. Parabolic ring face encourages the thicker film thickness at bearing pair interface (Morris et al., 2013b). The higher crown height or steep converging and diverging face, results in imminent hydrodynamic pressure drop and lower film thickness, which might encourage the mixed or boundary lubrication. In addition, high pressure is built on the converging part (leading edge) but generation of hydrodynamic pressure on divergent portion (trailing edge) of the ring is not an easy task. Therefore, the converging edge is responsible for the generated hydrodynamic pressure and governs the film thickness. Lower crown might encourage the generation of hydrodynamic pressure on trailing edge in comparison to higher crown height.

Figure 13 demonstrates the $h_{m}$ variation under mixed and hydrodynamic lubrication applying Reynolds cavitation and reformation boundary conditions at engine speed of $2000 \mathrm{rpm}$. There is no significant deviation of $h_{m}$ under both lubrication regimes, but under hydrodynamic lubrication, thicker oil film thickness has been obtained. Compared with oil separation and Reynolds cavitation and reformation boundary conditions under mixed lubrication, considering Sommerfeld solution results in thicker $h_{m}$ at ring/liner interface as shown in Fig. 14. Sommerfeld boundary conditions encourages larger effective ring width and area between oil layers to interact with each other (as oil flow is assumed to be laminar) which results in thicker film. Figure 15 compares the friction force under hydrodynamic and mixed regimes lubrication at 2000 rpm employing Reynolds cavitation and separation boundary conditions during numerical solution of Reynolds equation.

The higher the engine spin speed, the higher frictional loss as shown in Fig. 16. Figure 17(a) and (b) compare power loss under hydrodynamic and mixed lubrication against experiment data for alternative boundary conditions, half Sommerfeld, oil separation and Reynolds cavitation and separation. Due to lower deviation of calculated power loss under mixed lubrication from experimental data, it might be concluded that dominated regime during engine strokes is mixed lubrication. It was observed that considering oil separation boundary conditions results in lower deviation from experimental data, followed by Sommerfeld boundary conditions under mixed lubrication. 


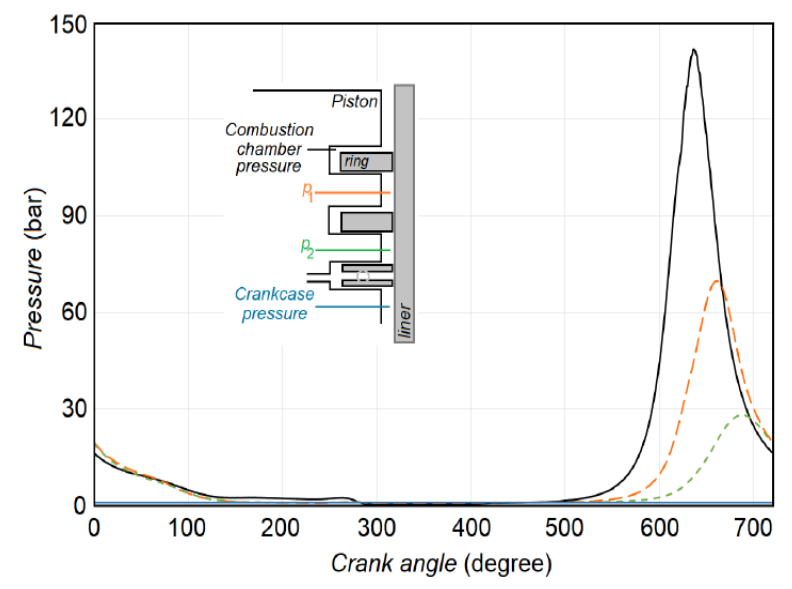

- Combustion chamber pressure $--p_{1}--p_{2}-$ Crankcase pressure

Fig. 4: Inter ring gas pressure at spin speed of $2000 \mathrm{rpm}$

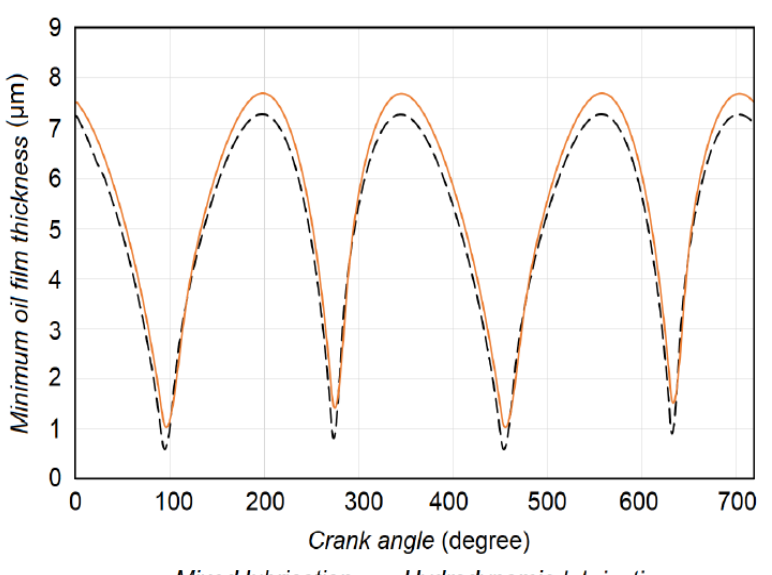

Fig. 5: Minimum oil film thickness applying half sommerfeld boundary conditions

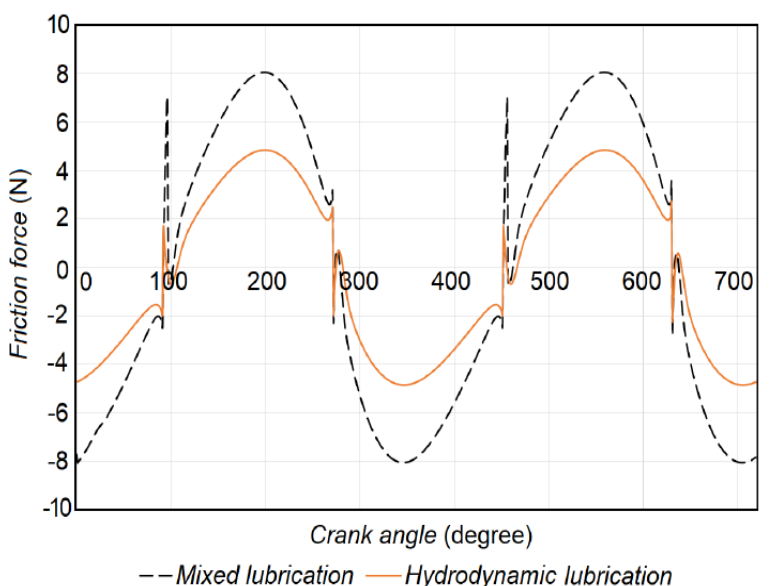

Fig. 6: Friction force applying half sommerfeld boundary conditions

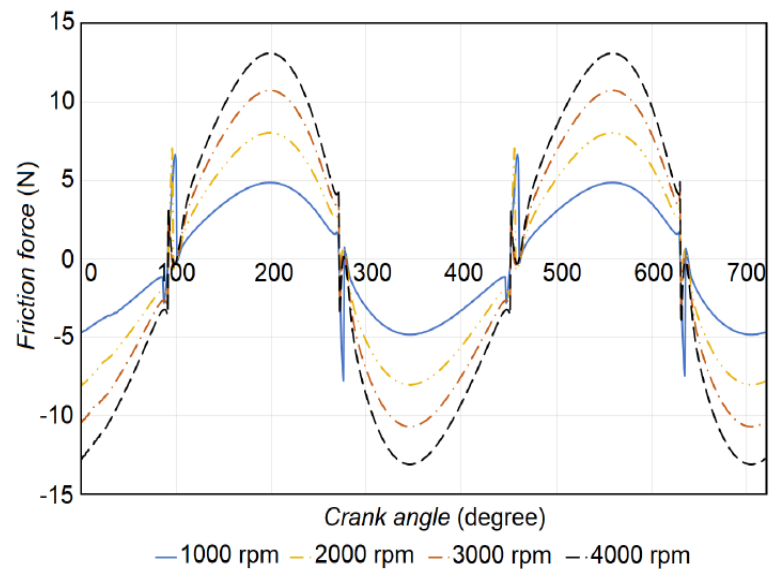

Fig. 7: Friction force applying half Sommerfeld boundary conditions at different engine spin speed

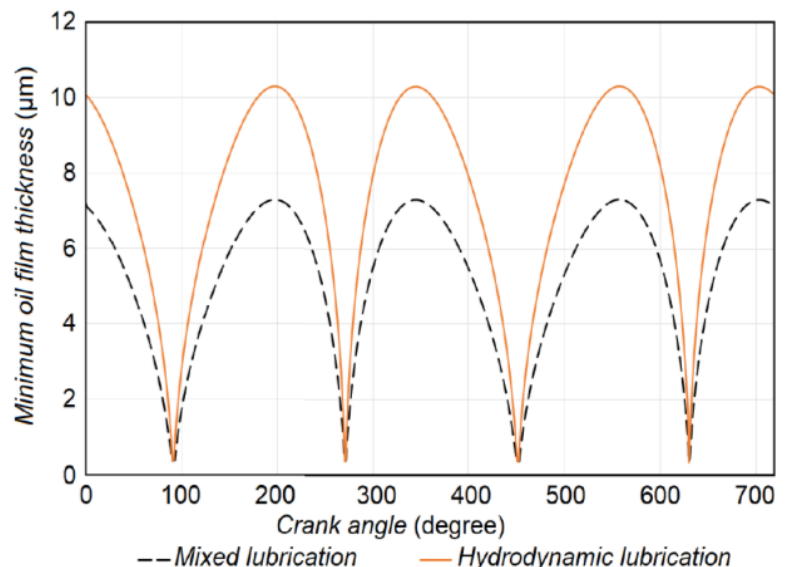

Fig. 8: Minimum oil film thickness applying oil separation boundary conditions

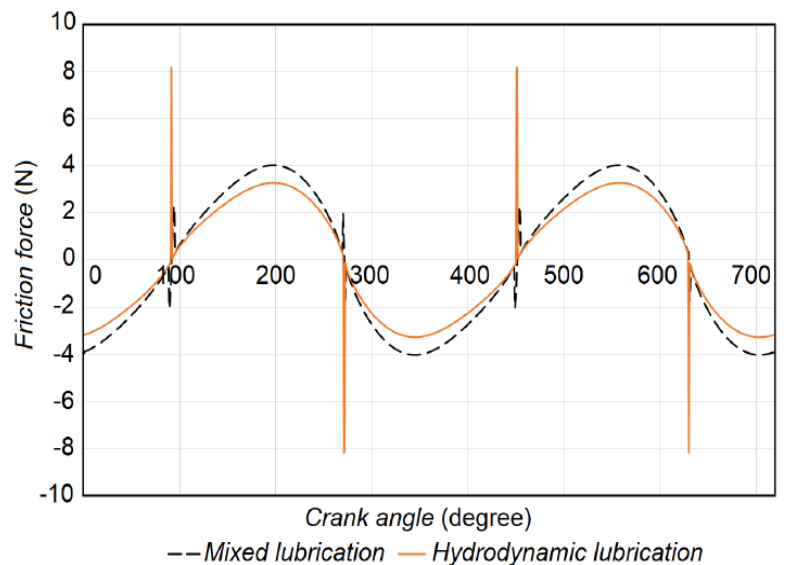

Fig. 9: Friction force applying oil separation boundary conditions 


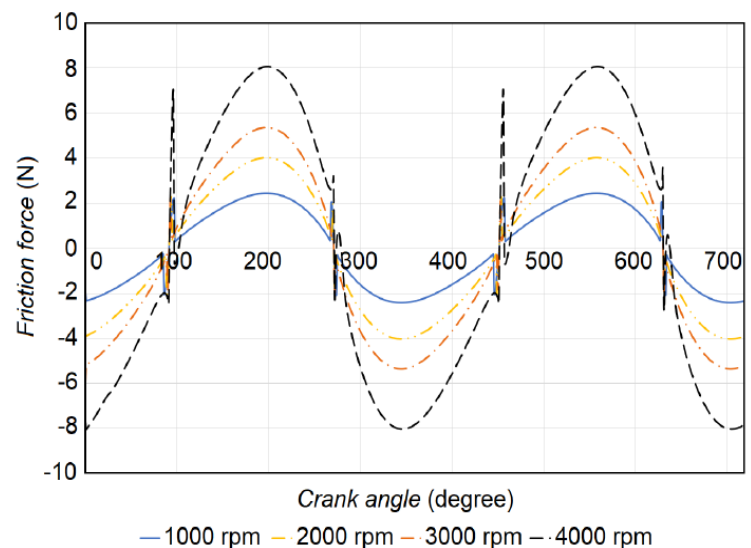

Fig. 10: Friction force applying oil separation boundary conditions at different engine spin speed

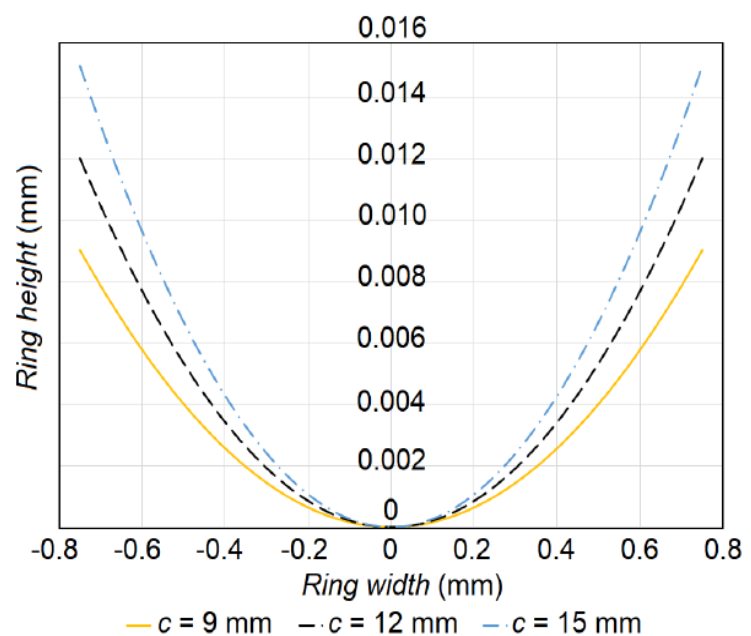

Fig. 11: Ring axial profiles considering different crown height

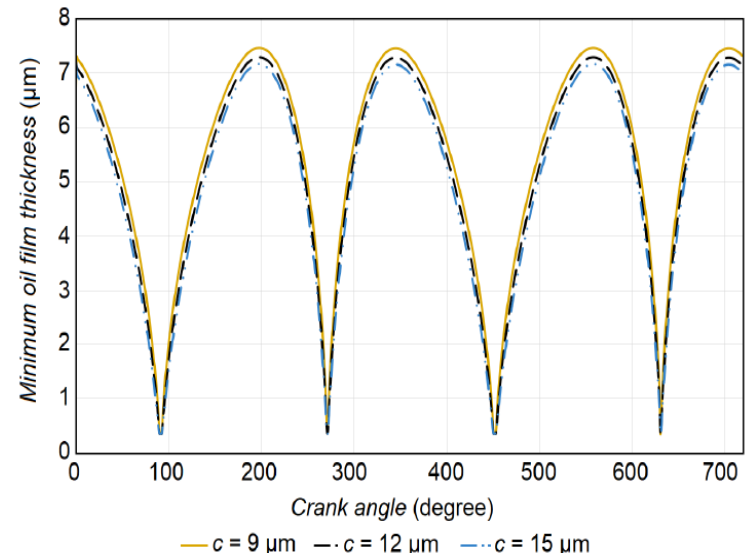

Fig. 12: Minimum oil film thickness considering different crown height

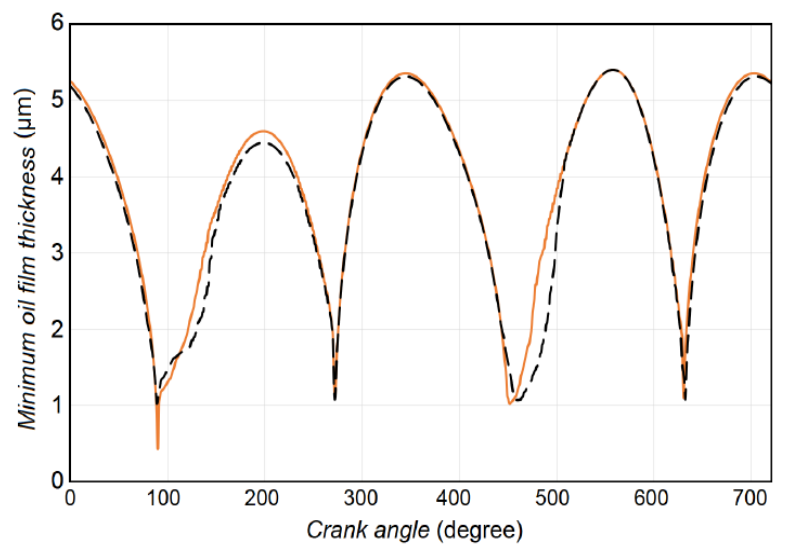

-- Mixed lubrication —-Hydrodynamic lubrication

Fig. 13: Minimum oil film thickness applying reynolds cavitation and reformation boundary conditions

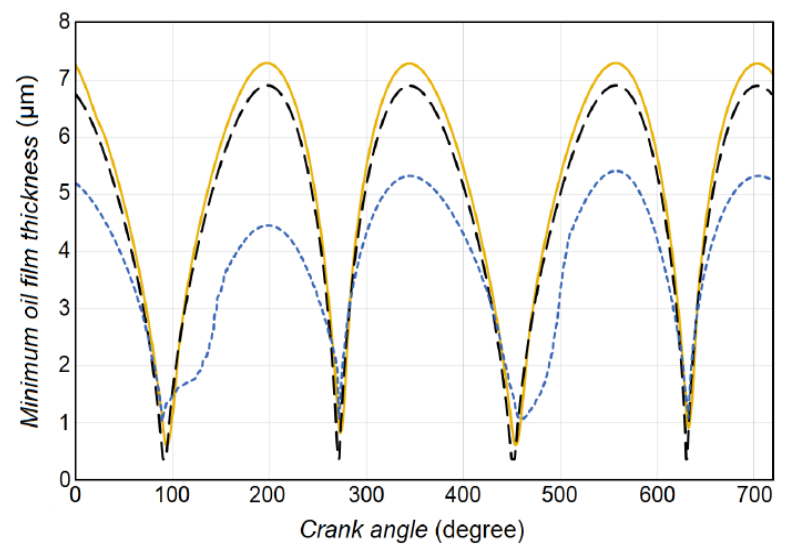

-Half Sommerfeld -- Oil separation --- Reynolds cavitation and reformatic

Fig. 14: The impact of boundary conditions type on minimum oil film thickness at engine spin of $2000 \mathrm{rpm}$

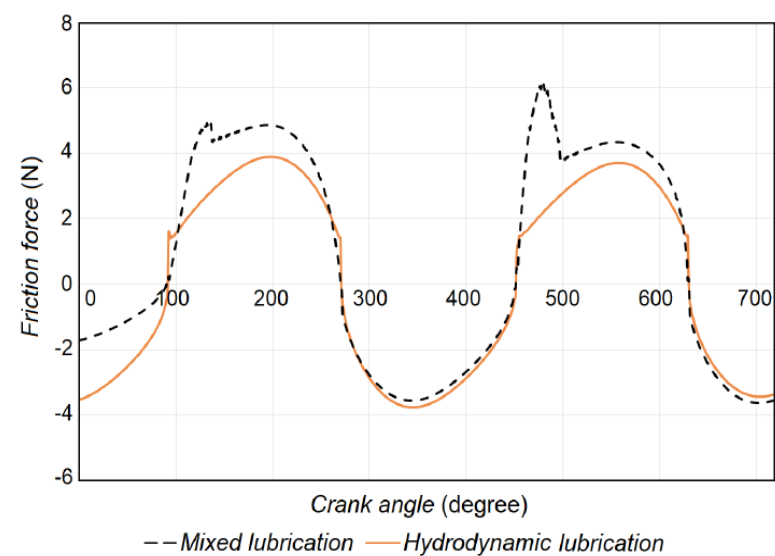

Fig. 15: Friction force applying Reynolds cavitation and separation boundary conditions 


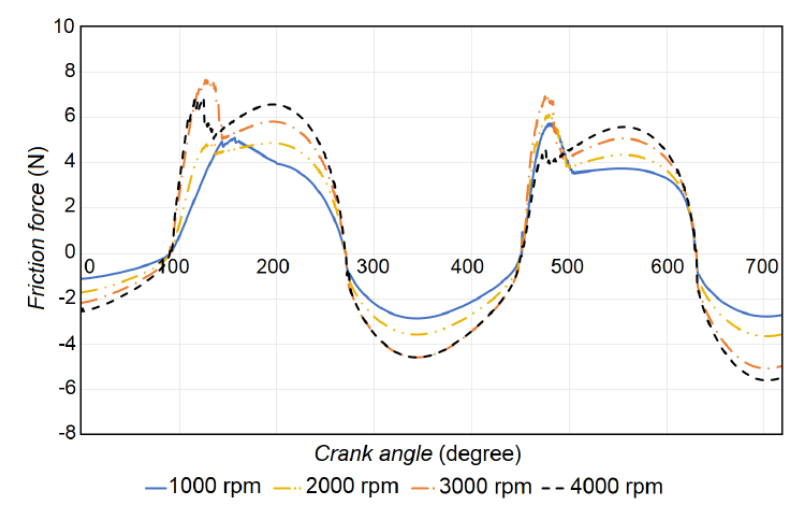

Fig. 16: Friction force applying Reynolds cavitation and reformation boundary conditions at different engine spin speed
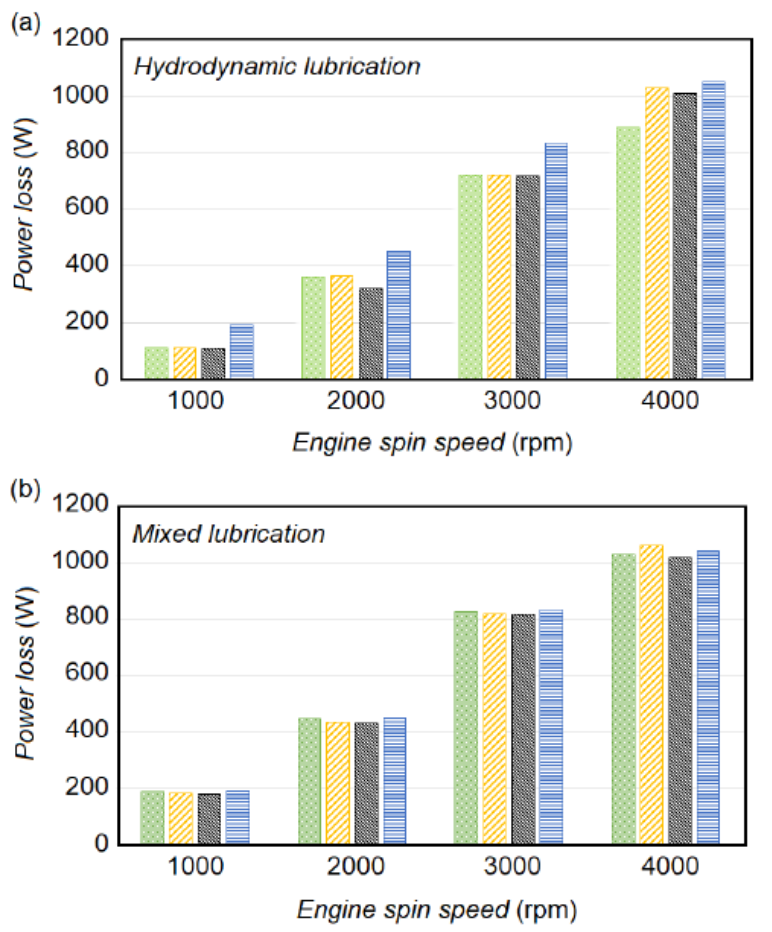

Half Sommerfeld boundary conditions

- Reynolds cavitation and reformation boundary conditions

Oil separation boundary conditions

亩 Experiment

Fig. 17: Power loss (a) hydrodynamic and (b) mixed lubrication regimes compared against experimental results applying Sommerfeld, oil separation and Reynolds cavitation and reformation boundary conditions

\section{Conclusion}

The numerical solution of Reynolds equation to govern the lubrication of piston ring/liner interface are sensitive to the assumed boundary conditions. Oil film shape, thickness and the nature of generated hydrodynamic pressure are under influence of such sensitivity. This study evaluates the effects of alternative boundary conditions, half Sommerfeld, oil separation and Reynolds cavitation and reformation conditions on piston ring tribology under isothermal mixed and hydrodynamic lubrication regimes. The current investigation depicts that ring/liner junction lubrication, oil film shape and tribology are under influence of lubrication regime and considered boundary conditions to solve Reynolds equation. Thicker oil film thickness under both hydrodynamic and mixed lubrication regimes has been calculated applying half Sommerfeld boundary conditions followed by oil separation due to larger effective of the ring width. The larger the effective ring width, the lower the normal load and in consequence thicker film thickness. As the numerical results under mixed lubrication deviates from experimental data less than hydrodynamic lubrication, it might be concluded that piston ring mostly enjoys mixed lubrication during engine strokes. It was observed that applying oil separation boundary conditions provides lower deviation from experimental data.

\section{Author's Contributions}

Abbas Razavykia: Analytical modeling and numerical simulation and developing MATLAB codes.

Cristiana Delprete: Conception and design and acquisition of data developed and verify the formulation.

Eugenio Brusa: Supervised experimental trials and finalized the research to be submitted.

Yaser Hosseini: Writing manuscript and preparing test bench to conduct experimental trials and validation the numerical results.

\section{Ethics}

This article is original and contains unpublished material. The corresponding author confirms that all of the other authors have read and approved the manuscript and no ethical issues involved.

\section{References}

Akalin, O., \& Newaz, G. M. (2001). Piston ring-cylinder bore friction modeling in mixed lubrication regime: part I-analytical results. J. Trib., 123(1), 211-218. doi.org/10.1115/1.1286337

Cho, S. W., Choi, S. M., \& Bae, C. S. (2000). Frictional modes of barrel shaped piston rings under flooded lubrication. Tribology International, 33(8), 545-551. doi.org/10.1016/S0301-679X (00)00103-1

Chu-Jung, H., \& Cheng, W. (1991). Elastohydrodynamic lubrication of piston rings. Wear, 150(1-2), 203-215. doi.org/10.1016/0043-1648(91)90317-N 
Delprete, C., \& Razavykia, A. (2018). Piston ring-liner lubrication and tribological performance evaluation: a review. Proceedings of the institution of mechanical engineers, part $\mathrm{J}$ : journal of engineering tribology, 232(2), 193-209. doi.org/10.1177/1350650117706269

Delprete, C., Razavykia, A., \& Baldissera, P. (2020). Detailed analysis of piston secondary motion and tribological performance. International Journal of Engine Research, 21(9), 1647-1661. doi.org/10.1177/1468087419833883

Dowson, D., Ruddy, B. L., \& Economou, P. N. (1983). The elastohydrodynamic lubrication of piston rings. Proceedings of the Royal Society of London. A. Mathematical and Physical Sciences, 386(1791), 409-430. doi.org/10.1098/rspa.1983.0043

Greenwood, J. A., \& Tripp, J. H. (1970). The contact of two nominally flat rough surfaces. Proceedings of the institution of mechanical engineers, 185(1), 625-633. doi.org/10.1243/PIME_PROC_1970_185_069_02

Guo, Y., Lu, X., Li, W., He, T., \& Zou, D. (2015). A mixedlubrication model considering elastoplastic contact for a piston ring and application to a ring pack. Proceedings of the Institution of Mechanical Engineers, Part D: Journal of Automobile Engineering, 229(2), 174-188. doi.org/10.1177/0954407014536149

Han, D. C., \& Lee, J. S. (1998). Analysis of the piston ring lubrication with a new boundary condition. Tribology international, 31(12), 753-760. doi.org/10.1016/S0301-679X (98)00096-6

Hu, Y. Z., \& Zhu, D. (2000). A full numerical solution to the mixed lubrication in point contacts. J. Trib., 122(1), 1-9. doi.org/10.1115/1.555322

Jeng, Y. R. (1992). Theoretical analysis of piston-ring lubrication Part I-fully flooded lubrication. Tribology transactions, 35(4), 696-706. doi.org/10.1080/10402009208982174

Livanos, G., \& Kyrtatos, N. P. (2006). A model of the friction losses in diesel engines (No. 2006-01-0888). SAE Technical Paper. doi.org/10.4271/2006-01-0888

Mishra, P. C., Rahnejat, H., \& King, P. D. (2009). Tribology of the ring-bore conjunction subject to a mixed regime of lubrication. Proceedings of the Institution of Mechanical Engineers, Part C: Journal of Mechanical Engineering Science, 223(4), 987-998. doi.org/10.1243/09544062JMES1220
Morris, N., Rahmani, R., Rahnejat, H., King, P. D., \& Fitzsimons, B. (2013a). Tribology of piston compression ring conjunction under transient thermal mixed regime of lubrication. Tribology International, 59, 248-258. doi.org/10.1016/j.triboint.2012.09.002

Morris, N., Rahmani, R., Rahnejat, H., King, P. D., \& Fitzsimons, B. (2013b). The influence of piston ring geometry and topography on friction. Proceedings of the Institution of Mechanical Engineers, Part J: Journal of Engineering Tribology, 227(2), 141-153. doi.org/10.1177/1350650112463534

Patir, N., \& Cheng, H. S. (1979). Application of average flow model to lubrication between rough sliding surfaces. doi.org/10.1115/1.3453329

Priest, M., Dowson, D., \& Taylor, C. M. (2000). Theoretical modelling of cavitation in piston ring lubrication. Proceedings of the Institution of Mechanical Engineers, Part C: Journal of Mechanical Engineering Science, 214(3), 435-447. doi.org/10.1243/0954406001523092

Priest, M., Taylor, R. I., Dowson, D., \& Taylor, C. M. (1996). Boundary conditions for Reynolds equation with particular reference to piston ring lubrication. In Tribology series (Vol. 31, pp. 441-452). Elsevier. doi.org/10.1016/S0167-8922(08)70806-X

Rahmani, R., Rahnejat, H., Fitzsimons, B., \& Dowson, D. (2017). The effect of cylinder liner operating temperature on frictional loss and engine emissions in piston ring conjunction. Applied energy, 191, 568581. doi.org/10.1016/j.apenergy.2017.01.098

Richardson, D. E., \& Borman, G. L. (1992). Theoretical and experimental investigations of oil films for application to piston ring lubrication (No. 922341). SAE Technical Paper. doi.org/10.4271/922341

Taylor, R. I. (2015). Squeeze film lubrication in piston rings and reciprocating contacts. Proceedings of the Institution of Mechanical Engineers, Part J: Journal of Engineering Tribology, 229(8), 977-988. doi.org/10.1177/1350650114564234

Wu, C., \& Zheng, L. (1989). An average Reynolds equation for partial film lubrication with a contact factor. doi.org/10.1115/1.3261872S 